\title{
Novel MRI Methodology to detect Human Whole-Brain Connectivity Changes after Ingestion of Fructose or Glucose
}

\author{
Sinchai Tsao ${ }^{a}$ Bryce Wilkins $^{a}$ Kathleen A. Page $^{b}$ and Manbir Singh ${ }^{a, c}$ \\ ${ }^{a}$ Department of Biomedical Engineering, Viterbi School of Engineering, \\ University of Southern California, Los Angeles, California, USA \\ ${ }^{b}$ Department of Internal Medicine, Division of Endocrinology, Keck School of Medicine, \\ University of Southern California, Los Angeles, California, USA \\ ${ }^{c}$ Department of Radiology, Keck School of Medicine, \\ University of Southern California, Los Angeles, California, USA
}

\begin{abstract}
A novel MRI protocol has been developed to investigate the differential effects of glucose or fructose consumption on whole-brain functional brain connectivity. A previous study has reported a decrease in the fMRI blood oxygen level dependent (BOLD) signal of the hypothalamus following glucose ingestion, but due to technical limitations, was restricted to a single slice covering the hypothalamus, and thus unable to detect whole-brain connectivity. In another previous study, a protocol was devised to acquire whole-brain fMRI data following food intake, but only after restricting image acquisition to an MR sampling or repetition time (TR) of 20s, making the protocol unsuitable to detect functional connectivity above $0.025 \mathrm{~Hz}$. We have successfully implemented a continuous 36-min, 40 contiguous slices, whole-brain BOLD acquisition protocol on a $3 \mathrm{~T}$ scanner with $\mathrm{TR}=4.5 \mathrm{~s}$ to ensure detection of up to $0.1 \mathrm{~Hz}$ frequencies for whole-brain functional connectivity analysis. Human data were acquired first with ingestion of water only, followed by a glucose or fructose drink within the scanner, without interrupting the scanning. Whole-brain connectivity was analyzed using standard correlation methodology in the 0.01-0.1 $\mathrm{Hz}$ range. The correlation coefficient differences between fructose and glucose ingestion among targeted regions were converted to t-scores using the water-only correlation coefficients as a null condition. Results show a dramatic increase in the hypothalamic connectivity to the hippocampus, amygdala, insula, caudate and the nucleus accumben for fructose over glucose. As these regions are known to be key components of the feeding and reward brain circuits, these results suggest a preference for fructose ingestion.
\end{abstract}

Keywords: MRI, fMRI, Functional Connectivity, Glucose, Fructose, Obesity, Insulin, Hypothalamus

\section{INTRODUCTION}

The main objective of these pilot studies was to develop a MRI protocol and conduct initial evaluation studies of the differential effects of glucose and fructose ingestion on the feeding pathways in normal (healthy) and obese adults. By identifying brain regions that show differential activity across normal and obese subjects following glucose or fructose ingestion, we hope that the data will permit new understandings of the effects of glucose/fructose ingestion and their implication in obesity. Recent published research ${ }^{1-4}$ has shown that glucose ingestion in healthy humans leads to a decrease in hypothalamic nuclei activity and consequently a decrease in the fMRI blood oxygen level dependent (BOLD) signal. Furthermore, it has been shown that glucose ingestion fails to inhibit hypothalamic nuclei activity in patients with type 2 diabetes. ${ }^{5}$ These scans typically require continuous acquisition of fMRI data for a period of about 30-45 minutes, and due to MRI technical limitations, previous research in this area has been restricted to acquisition of fMRI data from a single midsagittal slice, covering only the hypothalamus. A fundamental difference in our proposed studies was to design a protocol to obtain fMRI sampling over the entire brain, permitting the detection of glucose/fructose related changes throughout the brain. A successful protocol would then enable us to conduct an analysis of whole-brain functional connectivity following glucose/fructose ingestion. To our knowledge, no such connectivity analysis has been reported previously by any group. Thus, development of a MRI scan protocol for this project, which presents many key technological challenges, was considered an essential first step.

Further author information: (Send correspondences to Sinchai Tsao (stsao@usc.edu)) 


\section{METHODS}

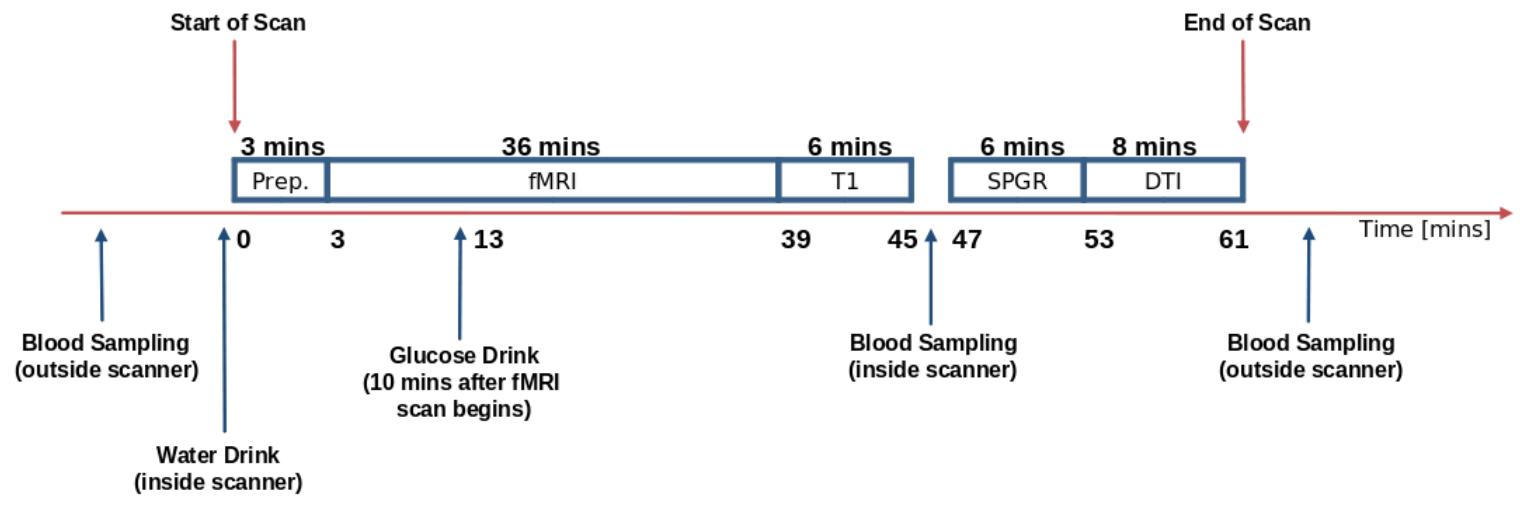

Figure 1. Three blood samples are taken during the experiment to track the subjects blood sugar level. The sequence of the individual scans, and times at which blood-sampling occurs, are indicated in the figure above.

\subsection{SUBJECTS}

This protocol was tested with 4 Hispanic subjects between the ages of 15-25 who have no history of diabetes.

\subsection{PROTOCOL DEVELOPMENT}

The challenge was to develop a whole-brain fMRI protocol with continuous data acquisition for approximately 45 minutes that would include a baseline, a relatively long post-glucose/fructose ingestion period, and a short time for ingesting the glucose/fructose during the scan. Typically a TR of 2 seconds is used in fMRI connectivity studies and for $\mathrm{TR}=2 \mathrm{~s}$, continuous acquisition of $4 \mathrm{~mm}$ thick images covering the whole brain would require a time-series of 40,500 images. As such long time-series have never been reported before in fMRI and are not allowed by hardware constraints of many scanners, we did several developmental scans to push the technical limits of the USC GE 3T MRI to increase the number of images that could be acquired in a continuous timeseries. It was found that our $3 \mathrm{~T}$ system could acquire at most 20,000 images continuously before exhausting its current memory capabilities. However during some developmental scans, the gradients overheated and the scans had to be terminated.

We argued that as glucose/fructose were diluted in water during ingestion, it would be important to add a baseline study corresponding to ingestion of water only, so that confounding effects of water could be separated from those attributed to sugar ingestion. We conducted several developmental studies to assess the fMRI signals acquired with water ingestion only and those after ingestion of a sugar drink and came to the conclusion that because all images must be acquired continuously (stopping and restarting a scan is not an option as it changes many parameters including the baseline making an accurate analysis virtually impossible) it was essential to acquire data from a water only period prior to sugar ingestion in the same continuous scan. Incorporating these requirements, we came up with our final 36 min fMRI protocol during which the subject first drinks pure water, followed by $10 \mathrm{~min}$ of data acquisition, then drinks water containing glucose (or fructose), followed by about 24 min of data acquisition. Thus the fMRI scan was divided into three regions: a 10 min baseline condition before ingestion of the sugar drink; drink period of 2-4 min; and lastly 22-24 min of post-drink data (see Figure 1). After several trials, the repetition time (TR) of the fMRI scan was chosen to be 4.5s. This TR is short enough for adequate temporal filtering of the data for functional connectivity analysis, and long enough to enable us to acquire data continuously for $36 \mathrm{~min}$ for a total of approximately 19,000 fMRI images. As additional images and time are required for anatomical and diffusion tensor imaging (DTI) analysis, 36 minutes were considered as close to the upper limit for the fMRI portion to finish the entire scan in about one hour.

Immediately following the fMRI acquisition, an anatomical T1-weighted scan is acquired to enable registration of subject fMRI data to an anatomical reference template. This is followed by another higher-resolution (SPGR) 
anatomical scan that is extensively used to spatially normalize images and delineate small anatomical regions of interest such as the hypothalamus.

Following the anatomical scans a diffusion-weighted data set is obtained in accordance with a standard DTI protocol that we have implemented on this GE scanner. This data may be used to investigate structural connectivity differences between normal (healthy) and obese subjects.

\subsection{DATA PROCESSING AND ANALYSIS OF fMRI BASELINE TRENDS}

As subjects are bound to move their heads during a relatively long scan and certainly during the drinking period, the first step in processing is to correct the fMRI time-series for head-movements. Each subjects' fMRI data is motion-corrected to the last acquired head-volume, registered to the individuals' high-resolution SPGR anatomical scan, and warped to normalized space through non-linear mapping techniques. The Colin Brain template $^{6}$ in Talairach Tournoux coordinates was used to define normalized space in this study though other templates such as the SPM MNI template could also be used. Regions of Interest (ROIs) are derived from the San Antonio Talairach Daemon, ${ }^{7,8}$ which is also in the Talairach-Tournoux normalized space.

\subsection{CONNECTIVITY ANALYSIS}

Whole-brain connectivity was analyzed using a standard technique where the movement and slice-timing corrected fMRI data are band-pass filtered $\left(\begin{array}{lll}0.01 & 0.10 \mathrm{~Hz}\end{array}\right)$, a seed region is selected and then the time courses of all voxels throughout the brain volume are correlated to the time-course of the seed region to obtain a measure of functional connectivity. An alternative approach is to first define ROIs based on anatomy and then compute the pair-wise connectivity among averaged time-courses of these ROIs. Both methods were pursued. In addition, the resting state default connectivity network was also examined. A methodology was developed to answer the following questions: a) Is there a significant difference between the resting state functional connectivity of the default mode network, which generally shows strong functional connectivity among the posterior cingulate and certain parietal and frontal regions, after ingesting glucose vs. fructose? b) Is there a significant difference between the whole-brain functional connectivity of the hypothalamus after ingesting glucose vs. fructose? c) Is there a significant difference in the pair-wise functional connectivity among specified ROIs after ingesting glucose vs. fructose? To reduce the effects of inter-subject variations, a pair-wise connectivity analysis was conducted after averaging the time-courses in targeted ROIs of 3 subjects who completed the glucose and fructose scans. The fourth subjects drinking times were significantly different to other subjects and as a result data from this subject was not included in the pair-wise connectivity analysis. The selected ROIs included key brain regions previously suggested to be involved in brain feeding and reward circuits. The pair-wise connectivity scores were converted to t-scores by comparing to the water-only baseline connectivities of the same pairs.

\section{RESULTS}

An example of raw fMRI results from a single subject are shown in Figure 2. Due to scanner drift, these signal cannot be interpreted directly. To account for scanner drift we demeaned the signal as shown in Figure 3. Using a seed region and time course of the signal before the ingestion of glucose / fructose in the posterior cingulum we were able to derive correlation maps for the default mode / resting state network (see Figure 4 ). After the ingestion of glucose / fructose we again used the same seed region and we were able to qualitative observe changes in the network (see Figure 5). We repeated the same procedure but used the hypothalamus as the seed regions in pre-glucose/pre-fructose and post-glucose/post-fructose (see Figure 6). To expand the search beyond manual selection of seed regions such as the posterior cingulum and the hypothalamus we used ROIs derived from the San Antonio Talairach Daemon ${ }^{7,8}$ and correlated each of the ROIs with each other as shown in Figure 7. The colors on the matrix grid indicate the degree of correlation between the two ROIs. ROIs with t-scores $>$ 2.00 are shown in Table 1. 

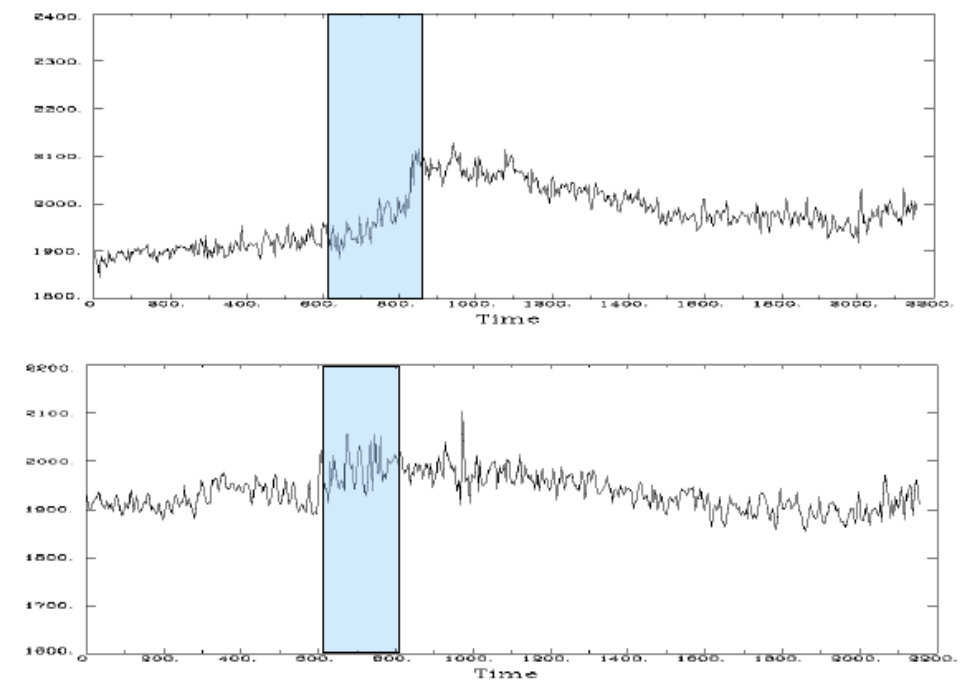

Figure 2. The hypothalamic fMRI time-course for a subject showing an initial water-only drink period, the actual drinking period (shaded) followed by either a glucose (top) or fructose (bottom) drink period. Glucose and fructose experiments were done on separate days.
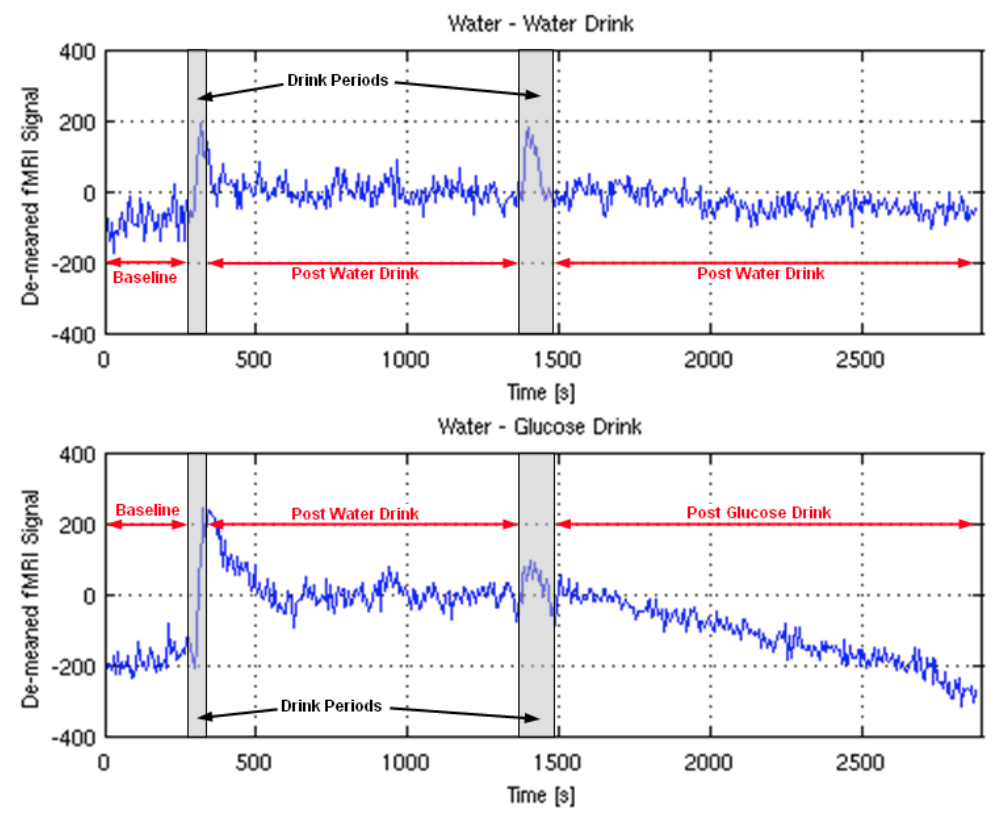

Figure 3. (top) The hypothalamic fMRI time-course includes a pre-drink baseline period preceding a water-only drink and then a second water-only drink. The data is de-trended by the first water-only period. (bottom) Same as top except the subject ingested a glucose drink during the second drinking period. The change in baseline trend following glucose ingestion is obvious. 

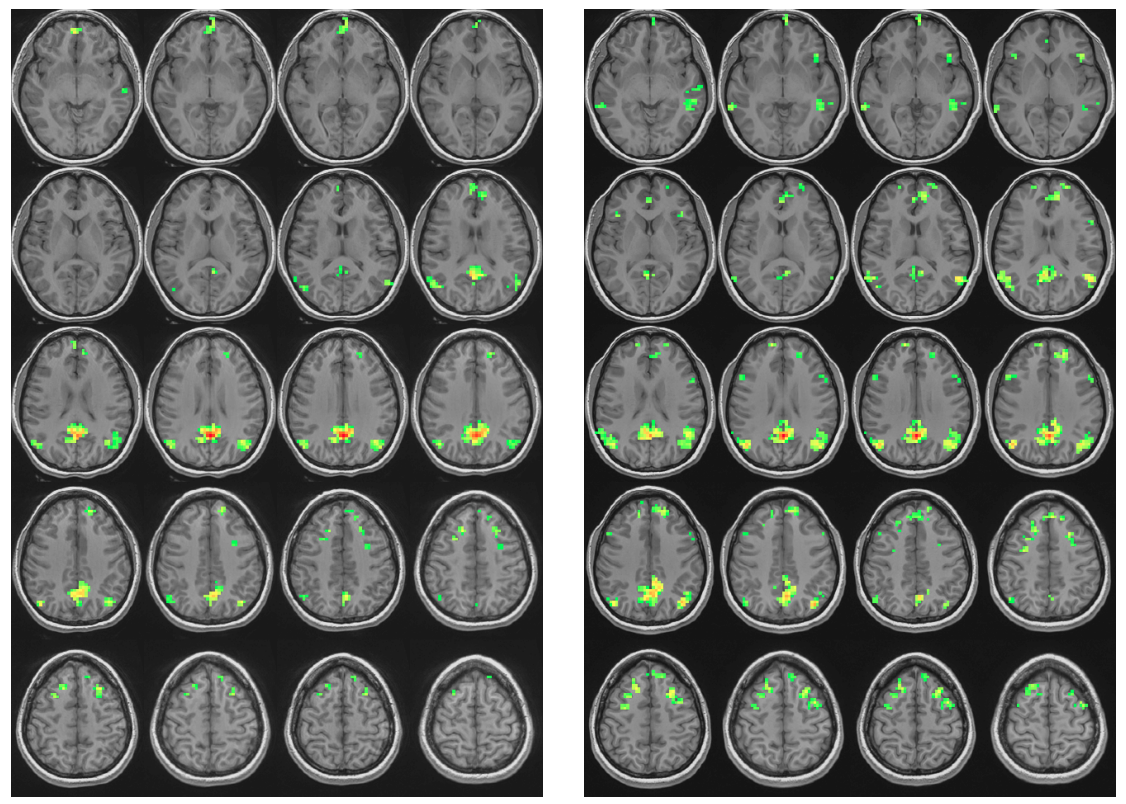

Figure 4. The default-mode network in a pilot subject during the pre-glucose (left) or the pre-fructose (right) period. These are expected to be the same but as the glucose and fructose experiments were done on separate days, minor differences can be seen. The default network is clearly identified by our protocol.
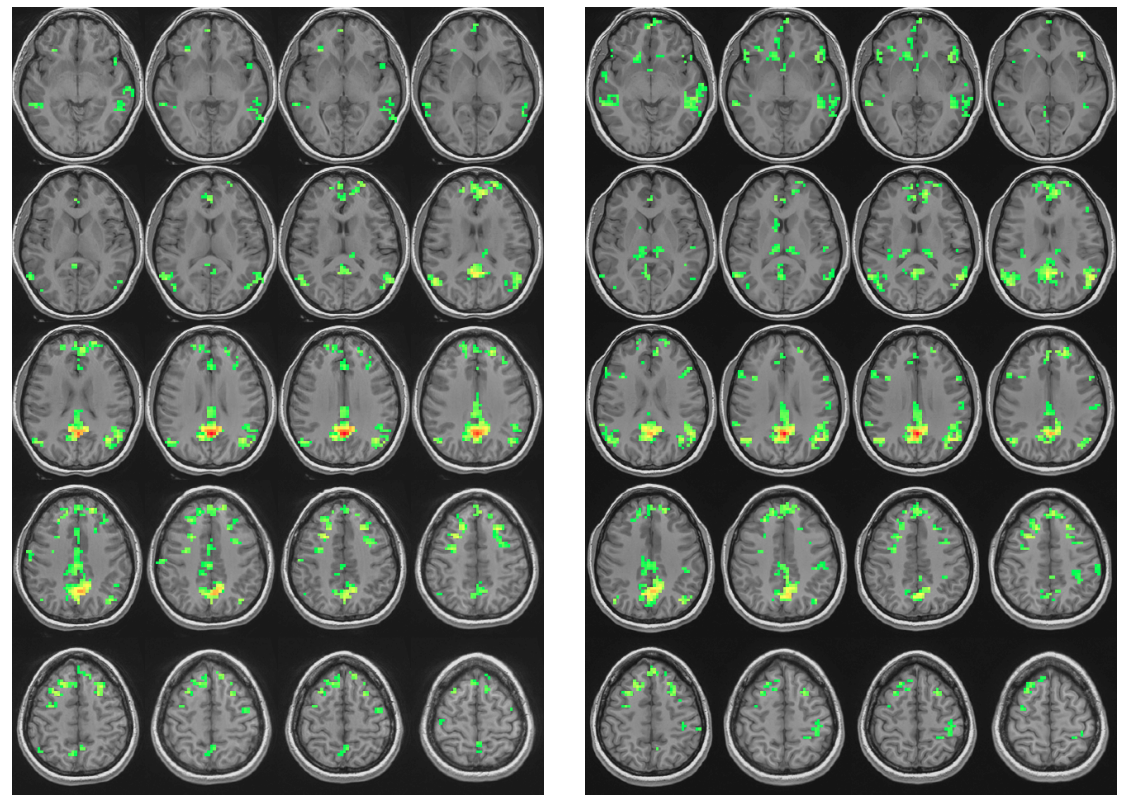

Figure 5. The default-mode network in a pilot subject during the post-glucose (left) or the post-fructose (right) period. Several differences can be seen, which need further analysis to interpret their significance. 

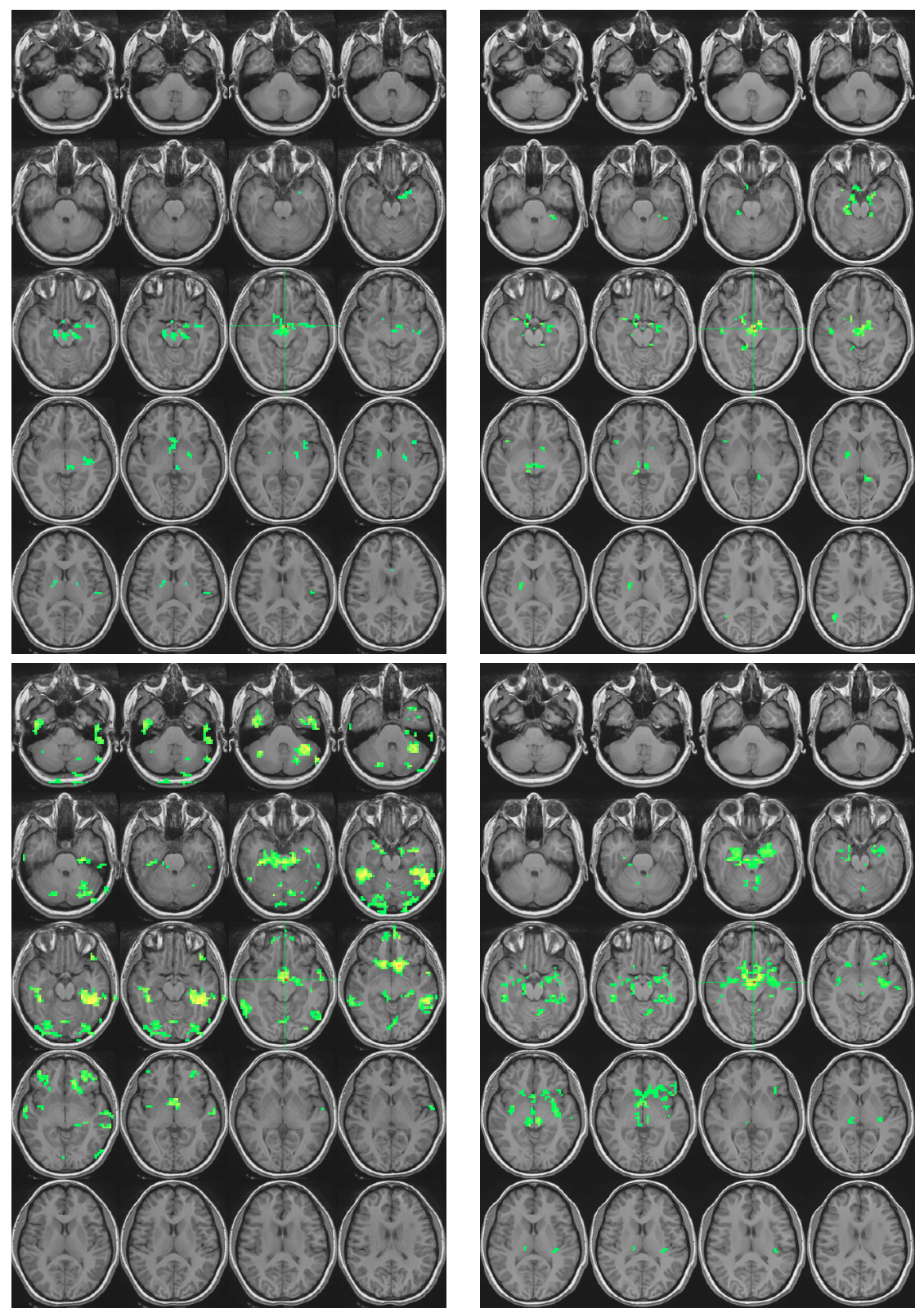

Figure 6. The hypothalamic connectivity in a pilot subject during the pre and post-glucose periods (top and bottom left respectively) or the pre and post-fructose periods (top and bottom right respectively). 


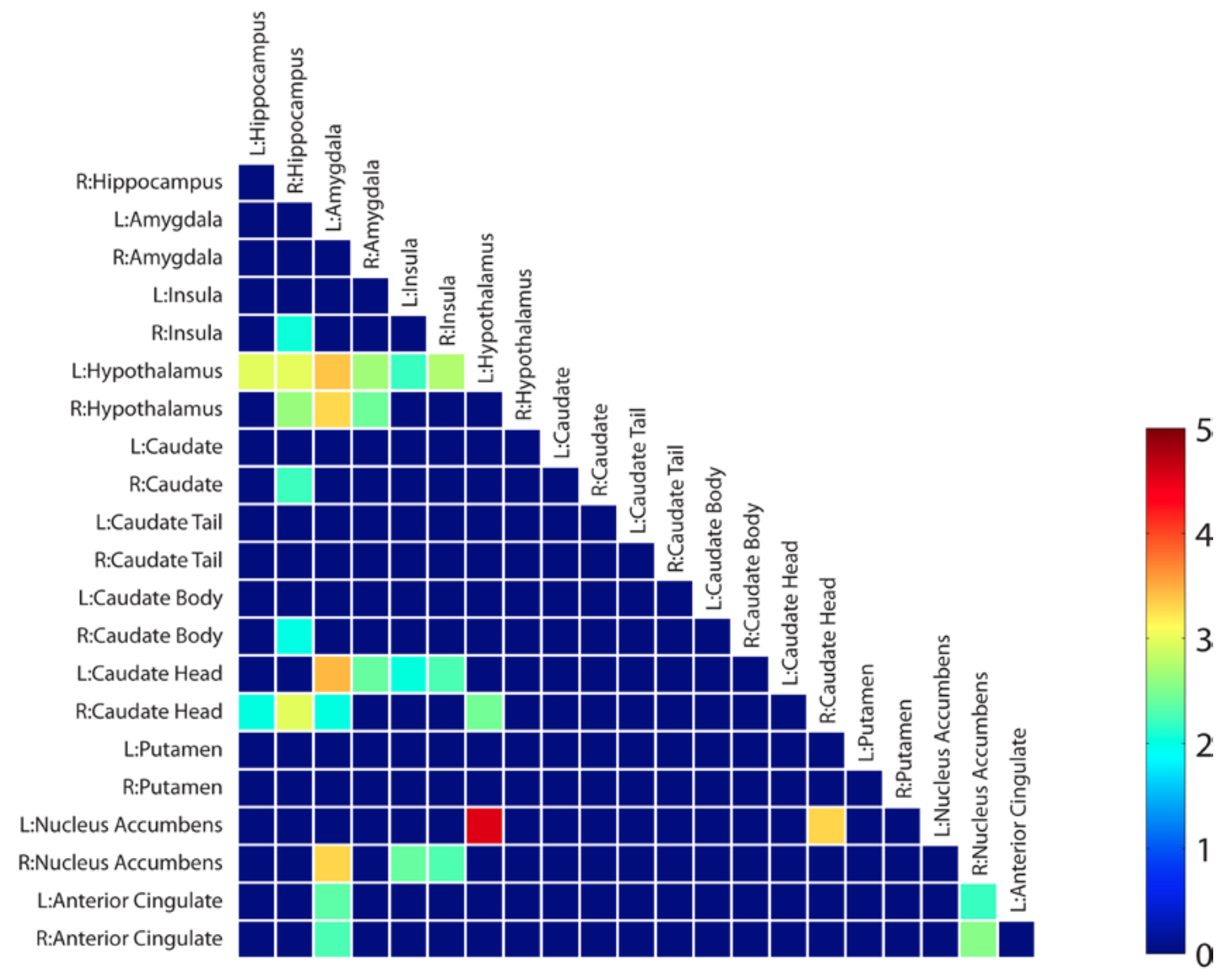

Figure 7. Matrix of thresholded t-scores $(t>2)$ from pair-wise functional connectivity between select ROIs averaged over 3 subjects; the color-bar gives the t-score to color mapping. The most prominent connectivity exists between the left hypothalamus and left/right hippocampus, left/right amygdala, left/right insula, right caudate head, and left nucleus accumbens. The highest t-score (4.57) is between the left hypothalamus and left nucleus accumbens. 
Table 1. t-scores corresponding to the pair-wise ROIs shown in Figure 7 that exceed the threshold $(\mathrm{t}>2)$.

\begin{tabular}{|l|l|c||l|l|c|}
\hline ROI 1 & ROI 2 & t-Score & ROI 1 & ROI 2 & t-Score \\
\hline \hline L:Hippocampus & L:Hypothalamus & 2.97 & R:Insula & L:Caudate Head & 2.30 \\
L:Hippocampus & R:Caudate Head & 2.02 & R:Insula & R:Nucleus Accumbens & 2.31 \\
R:Hippocampus & R:Insula & 2.08 & L:Hypothalamus & L:Hippocampus & 2.97 \\
R:Hippocampus & L:Hypothalamus & 3.01 & L:Hypothalamus & R:Hippocampus & 3.01 \\
R:Hippocampus & R:Hypothalamus & 2.64 & L:Hypothalamus & L:Amygdala & 3.41 \\
R:Hippocampus & R:Caudate & 2.23 & L:Hypothalamus & R:Amygdala & 2.68 \\
R:Hippocampus & R:Caudate Body & 2.02 & L:Hypothalamus & L:Insula & 2.22 \\
R:Hippocampus & R:Caudate Head & 2.99 & L:Hypothalamus & R:Insula & 2.76 \\
L:Amygdala & L:Hypothalamus & 3.41 & L:Hypothalamus & R:Caudate Head & 2.46 \\
L:Amygdala & R:Hypothalamus & 3.31 & L:Hypothalamus & L:Nucleus Accumbens & 4.57 \\
L:Amygdala & L:Caudate Head & 3.47 & R:Hypothalamus & R:Hippocampus & 2.64 \\
L:Amygdala & R:Caudate Head & 2.03 & R:Hypothalamus & L:Amygdala & 3.31 \\
L:Amygdala & R:Nucleus Accumbens & 3.31 & R:Hypothalamus & R:Amygdala & 2.45 \\
L:Amygdala & L:Anterior Cingulate & 2.36 & R:Caudate & R:Hippocampus & 2.23 \\
L:Amygdala & R:Anterior Cingulate & 2.27 & R:Caudate Body & R:Hippocampus & 2.02 \\
R:Amygdala & L:Hypothalamus & 2.68 & L:Caudate Head & L:Amygdala & 3.47 \\
R:Amygdala & R:Hypothalamus & 2.45 & L:Caudate Head & R:Amygdala & 2.41 \\
R:Amygdala & L:Caudate Head & 2.41 & L:Caudate Head & L:Insula & 2.05 \\
L:Insula & L:Hypothalamus & 2.22 & L:Caudate Head & R:Insula & 2.30 \\
L:Insula & L:Caudate Head & 2.05 & R:Caudate Head & L:Hippocampus & 2.02 \\
L:Insula & R:Nucleus Accumbens & 2.41 & R:Caudate Head & R:Hippocampus & 2.99 \\
R:Insula & R:Hippocampus & 2.08 & R:Caudate Head & L:Amygdala & 2.03 \\
R:Insula & L:Hypothalamus & 2.76 & R:Caudate Head & L:Hypothalamus & 2.46 \\
\hline
\end{tabular}

\section{DISCUSSION}

Examples of the connectivity images obtained from one such subject are presented in Figures 4,5 showing potential differences in the default network between glucose and fructose ingestion. A correlation threshold of 0.5 was applied. Similar connectivity differences between the hypothalamic ROI and voxels throughout the brain were also noted. As the hypothalamus is composed of just a few voxels and is anatomically located in a region of the brain where susceptibility artifacts are strong, the signal-to-noise ratio in the time-course is not very high. Thus more variation is expected in the hypothalamic connectivity analysis than the default network. The hypothalamic connectivity images are shown in Figure 6. Overall it appears that connectivity of several regions is altered post-fructose vs. post-glucose but there were significant inter-subject variations (which is expected).

Though the sample size is very small, the results show a dramatic increase in the hypothalamic connectivity to very specific regions known to be key components of the feeding and reward circuits after fructose ingestion when compared to glucose ingestion. Specifically, an increase in hypothalamic connectivity to the hippocampus, amygdala, insula, caudate and the nucleus accumbens stands out. The largest increase (t-score of 4.5, which means an increase of 4.5 standard deviations with respect to glucose connectivity) is seen for the hypothalamicnucleus accumbens connection, which is known to be a key component of the feeding circuit. The selected ROIs and their pair-wise connectivity results are shown in Figure 7. The t-scores for all ROIs above the threshold of $t>2$ are listed in Table 1 . No significant increases in pair-wise connectivity among the selected ROIs were detected for glucose compared to fructose ingestion.

\section{CONCLUSION}

After the publication of Liu et al. 2000's Nature article, ${ }^{4}$ there has been a lot of interest in being able to investigate how brain activity is influenced by food. The tool of choice has been fMRI. Liu et al 2000 showed how the use of the raw fMRI signal without deconvolution of the Hemodynamic Response Function (HRF) can 
give us repeatable detection of temporal changes in brain activations even though responses from HRF-based analysis can be seen. ${ }^{9,10}$ Recent developments of functional connectivity and better knowledge about the default mode network has given us a new framework from which to investigate the basis of these changes. This paper outlines our initial efforts at attempting to investigate food related functional connectivity changes using the premise that the default mode network somehow modulates these responses.

We know that the default mode network is crucial in our ability to maintain homeostasis. ${ }^{11}$ Feeding behavior is one complex component of supplying nutrition to maintain homeostasis and therefore the default mode network should respond accordingly to food stimulus. The question is whether when and where do these stimuluses get translated into neurological pathways that solidify our behavior. In particular, how do these differences in default mode network responses reflect pathological states where the human body is unable to regulate feeding behavior properly, leading to the development of obesity. To make matters more complex, the causality is unknown. In other words, does the pathological feeding behavior cause the differences in responses or do the responses indicate an underlying condition that causes the pathological behavior. This coupled with the differential activations between fructose and glucose raises a large number of questions about the neurological mechanisms that govern our feeding behavior. To our knowledge, no such results have been reported before and if these results can be verified in a larger study, they would represent a key discovery in how brain connectivity among key regions is altered by fructose compared to glucose ingestion.

With regards to fMRI processing and techniques, this study serves to show that there is still much work that needs to be done to properly understand the results of fMRI studies. The underlying biological mechanisms that lead to the partial correlations in functional connectivity are not well know. ${ }^{11}$ Even if we were to be able to find changes that correlate well with parameters such as the ingestion of glucose versus fructose, we are not able to draw definitive conclusions unless the underlying mechanisms are better understood. Current glucose ingestion studies together with studies that use pharmaceuticals alter our homeostatic state ${ }^{12}$ will help to elucidate some of these mechanisms. This together with other anthropometric techniques as well as PET studies will further clarify the phenomenon observed in our fMRI studies. ${ }^{9,10}$ Techniques that better utilize the temporal information within the fMRI signal will continue to push the envelope in terms of the information elicited from imaging studies. Functional Connectivity and other techniques will continue to push the evolution of techniques that do not use the HRF. This will hopefully help us establish a better understanding of how each part of the brain responds vis-à-vis another part of the brain and not just be able to isolate individual activities as predicted by the BOLD HRF.

\section{ACKNOWLEDGMENTS}

The authors would like to acknowledge Samuel Valencerina for his help with the MRI at USC. Lab-mates Darryl Hwang for helping with the development of the MRI protocol. In particular, his help with the glucose ingestion delivery system. I'd like to dedicate this work to the late Professor Manbir Singh PhD, my mentor, friend and inspiration who's sudden passing this past winter has been a tragic loss for us all. This study was partially supported by National Center of Research Resources (NCRR) grant 1S10RR019942 that provided the 3T MRI used in this study.

\section{REFERENCES}

[1] Smeets, P. A. M., de Graaf, C., Stafleu, A., van Osch, M. J. P., and van der Grond, J., "Functional mri of human hypothalamic responses following glucose ingestion," Neuroimage 24, 363-8 (Jan 2005).

[2] Smeets, P. A. M., Vidarsdottir, S., de Graaf, C., Stafleu, A., van Osch, M. J. P., Viergever, M. A., Pijl, H., and van der Grond, J., "Oral glucose intake inhibits hypothalamic neuronal activity more effectively than glucose infusion," Am J Physiol Endocrinol Metab 293, E754-8 (Sep 2007).

[3] Vidarsdottir, S., Smeets, P. A. M., Eichelsheim, D. L., van Osch, M. J. P., Viergever, M. A., Romijn, J. A., van der Grond, J., and Pijl, H., "Glucose ingestion fails to inhibit hypothalamic neuronal activity in patients with type 2 diabetes," Diabetes 56, 2547-50 (Oct 2007).

[4] Liu, Y., Gao, J. H., Liu, H. L., and Fox, P. T., "The temporal response of the brain after eating revealed by functional mri," Nature 405, 1058-62 (Jun 2000). 
[5] Musen, G., Simonson, D. C., Bolo, N. R., Driscoll, A., Weinger, K., Raji, A., Théberge, J., Renshaw, P. F., and Jacobson, A. M., "Regional brain activation during hypoglycemia in type 1 diabetes," J Clin Endocrinol Metab 93, 1450-7 (Apr 2008).

[6] Holmes, C. J., Hoge, R., Collins, L., Woods, R., Toga, A. W., and Evans, A. C., "Enhancement of mr images using registration for signal averaging," J Comput Assist Tomogr 22(2), 324-33 (1998).

[7] Lancaster, J. L., Rainey, L. H., Summerlin, J. L., Freitas, C. S., Fox, P. T., Evans, A. C., Toga, A. W., and Mazziotta, J. C., "Automated labeling of the human brain: a preliminary report on the development and evaluation of a forward-transform method," Hum Brain Mapp 5(4), 238-42 (1997).

[8] Lancaster, J. L., Woldorff, M. G., Parsons, L. M., Liotti, M., Freitas, C. S., Rainey, L., Kochunov, P. V., Nickerson, D., Mikiten, S. A., and Fox, P. T., "Automated talairach atlas labels for functional brain mapping," Hum Brain Mapp 10, 120-31 (Jul 2000).

[9] Tsao, S., Adam, T. C., Goran, M. I., and Singh, M., "Application of fmri to obesity research: differences in reward pathway activation measured with fmri bold during visual presentation of high and low caloric foods," in [Proceedings of SPIE Medical Imaging, San Diego, CA], (2012).

[10] Stice, E., Spoor, S., Bohon, C., and Small, D. M., "Relation between obesity and blunted striatal response to food is moderated by taqia a1 allele," Science 322, 449-52 (Oct 2008).

[11] Snyder, A. Z. and Raichle, M. E., "A brief history of the resting state: The washington university perspective," Neuroimage (Jan 2012).

[12] Vidarsdottir, S., Vlug, P., Roelfsema, F., Frölich, M., and Pijl, H., "Orally disintegrating and oral standard olanzapine tablets similarly elevate the homeostasis model assessment of insulin resistance index and plasma triglyceride levels in 12 healthy men: a randomized crossover study," J Clin Psychiatry 71, 1205-11 (Sep 2010). 